\title{
Blood Pressure Models for Wearable Sensors
}

This paper was downloaded from TechRxiv (https://www.techrxiv.org).

\section{LICENSE}

CC BY 4.0

SUBMISSION DATE / POSTED DATE

29-07-2021 / 20-08-2021

\section{CITATION}

Gonçalves Seabra, Ana Carolina; Ferreira da Silva, Alexandre; Stieglitz, Thomas; Amado Rey, Ana Belen (2021): Blood Pressure Models for Wearable Sensors. TechRxiv. Preprint.

https://doi.org/10.36227/techrxiv.15073383.v2

$\mathrm{DOI}$ 


\title{
Blood Pressure Models for Wearable Sensors
}

\author{
A. C. Seabra, A. F. Silva, T. Stieglitz* Senior Member, IEEE, and A. B. Amado-Rey*
}

\begin{abstract}
As cardiovascular diseases are one of the most prominent illnesses, a continuous, non-invasive, and comfortable monitoring of blood pressure (BP) is indispensable. This paper investigates the best method for obtaining highly accurate BP values in non-invasive measurements when using an ultrasound (US) sensor projected for a wrist-worn device. State-of-the-art BP models were analyzed and qualitatively compared. Relevant arterial parameters such as luminal area, flow velocity and pulse wave velocity, of 729 subjects were extracted from a computer simulated database and served as input parameters for the wearable US. A linear in-silico model calibrated to each arterial-site revealed to be most accurate model. The linear model was used for the extraction of BP by using the US sensor and validated with a commercial pressure sensor in an ex-vivo experimental setup. The results showed an in-silico pulse pressure correlation of 0.978 and mean difference of $(-2.134 \pm 2.477) \mathrm{mmHg}$ at the radial artery and ex-vivo pressure correlation of 0.994 and mean difference of $(0.554 \pm 2.315)$ mmHg. Thus, with the linear model, the US measurement complies with the Association for the Advancement of Medical Instrumentation standard with deviations lower than $5 \mathrm{mmHg}$.

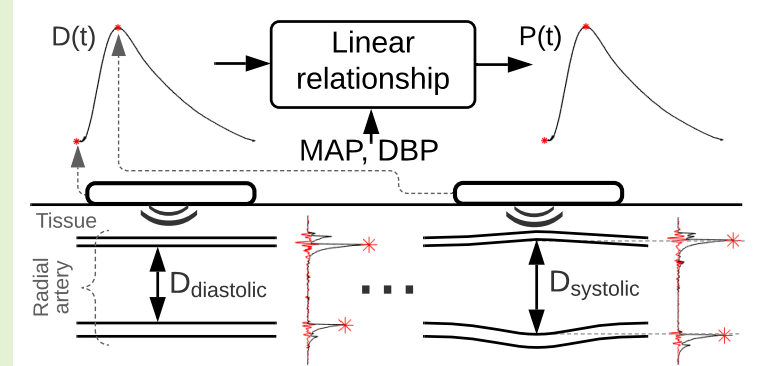

Index Terms - arterial pressure waveform, continuous and non-invasive BP measurement, hypertension monitoring, pulse pressure, ultrasound sensor, wearable devices.

\section{INTRODUCTION}

H YPERTENSION is the most common cause of cardiovascular diseases (CVD) worldwide. The higher the blood pressure (BP), the higher the risk of damage to the heart and blood vessels in the major organs. Hypertension is known as the "silent killer", because most people might even not show any symptoms at all, causing around half of all deaths from CVD [1]. Therefore, the need for continuous monitoring of BP in the daily life is evident. In a clinical context, hypertension can be diagnosed when multiple readings of systolic blood pressure $(S B P)$ and diastolic blood pressure $(D B P)$ are above $140 \mathrm{mmHg}$ and $90 \mathrm{mmHg}$, respectively [2]. Moreover, the BP pulse wave $\left(P W_{B P}\right)$ presents a wealth of information in the cardiovascular system, providing remarkable insights for CVDs diagnosis and prognosis at an early stage.

The gold standard for BP measurement is sphygmomanometry at the brachial artery, which besides being uncomfortable when used for long periods of time due to the periodic inflation

This work was submitted for review on August 6th 2021. The authors would like to thank Fraunhofer IBMT for providing the ultrasound sensor and B.Braun-Stiftung for funding this project (NINUSSense, BBST-D-19-00008).

* T. Stieglitz and A. B. Amado-Rey contributed equally to this work.

A. C. Seabra is with the Center for MicroEletroMechanical Systems (CMEMS-UMinho), University of Minho, Portugal and with the Department of Microsystems Engineering, University of Freiburg, Freiburg, Germany (correspondence e-mail: a80148@alunos.uminho.pt).

A. F. Silva is with the Center for MicroEletroMechanical Systems (CMEMS-UMinho), University of Minho, Portugal .

T. Stieglitz is with the Department of Microsystems Engineering, University of Freiburg, Germany

A. B. Amado-Rey is with the Department of Microsystems Engineering, University of Freiburg, Germany (e-mail: belen.amado@imtek.de). . 
than $15 \mathrm{mmHg}$ in comparison to the intra-brachial BP [12]. It is necessary to deduce an algorithm to continuously extract highly accurate SBP and DBP values from measurements in the radial artery.

This work performs an analytical analysis and comparative study between different models based on their physical principles, with their assumptions and simplifications explained. A computer-simulated arterial pulse wave (PW) database developed by Charlton et al. [13] was used to demonstrate how the pressure values vary between different arteries and to compare the response to the models at different measuring sites. In the in-silico study, accuracy of different mathematical models applied to the radial artery was investigated through parameters derived from the database. The best performing model was validated with a porcine artery and a customized ultrasound sensor. Data were post-processed in a custom analysis program written in the MATLAB environment. Finally, the accuracy of the ex-vivo measurements was studied through its comparison with a commercial sensor. The results showed promising advances and new insights for the extraction of vital parameters when using ultrasound sensors.

\section{Non-invasive Continuous BP Measurement}

\section{A. Tonometer Method}

The tonometer method is based on the measurement of back-force at contact when the device is placed over a superficial vessel. Applanation of the vessel wall requires a stiff or bony structure as abutment. The change in pressure of the flow is measured while the (static) pressure is applied. Controlled force has to be applied to avoid occlusion of the blood vessel. Thus, accurate positioning over the measuring site is required. Applanation tonometry cannot be applied to obese subjects, as it needs lean skin to avoid cushioning of the pressure values. This method is highly sensitive to motion, proving impractical for daily activities [3], [4].

\section{B. Vascular Unloading Method}

Also known as volume clamp method, vascular unloading method relies on a finger PPG sensor that adjusts pressure in a small finger cuff and keeps the optically measured blood flow constant. The varying adjusted cuff pressure can then be correlated to the intra-arterial BP. Continuous measurement of BP is possible with this method, but the cuff is uncomfortable for long periods of time and there is a risk of venous congestion for the patient [3]-[5].

\section{PTT Method}

PTT is the time it takes for a PW to travel between two points of the cardiovascular system. The configuration that gives the most accurate $S B P$ and $D B P$ values is the PTT obtained between an eletrocardiogram (ECG) and a finger PPG. The recorded signals can be translated to BP through a calibration procedure. Compared to the previously presented methods, PTT does not need to partly occlude a vessel, but comes with an increase in the system's complexity, as it adds a second PW measuring site [3], [14].

\section{Ultrasound-based Method}

Assessment of the BP waveform via ultrasound (US) technique utilizes a high-speed acoustic probe to capture the pulsation of arteries. On the one hand, echo signals and Doppler shift principle have been used in this technique to detect the arterial wall displacement and blood flow, which with a mathematical model and signal conditioning reveal good accuracy in arterial measurements and is, therefore, convenient to use. On the other hand, it is necessary to maintain a stable contact between the probe and the measuring site in order to achieve a reliable acoustic coupling interface [4], [8][11]. Taking the indicated advantages and disadvantages into account, the US-based method seems to be the most promising method, when stable acoustic coupling is achieved.

\section{Pressure Waveform Models}

For the application of a wrist wearable device to measure BP through an US-sensor, models that rely on the measurement of both hemodynamics (blood flow and arterial distension) of the arterial segment were analyzed and compared.

\section{A. Model 1 (M1): Linear Relationship}

Through a direct conversion, the diameter waveform, $D(t)$, can be projected to a pressure waveform [8]. The end-diastolic and mean arterial diameter, $D_{d}$ and $\bar{D}(t)$, are calibrated to the brachial $D B P$ and $M A P$, deriving the following conversion factor $k$ :

$$
k=\frac{M A P-D B P}{\bar{D}(t)-D_{d}} .
$$

The diameter waveform is then converted to the pressure waveform by the factor $k$ (1) and the slope intercept $b$ calculated at the diastolic pressure,

$$
P(t)=D(t) \cdot k+b .
$$

Although a study revealed that this method of assessing BP may on average only underestimate invasive $P P$ by $1.6 \mathrm{mmHg}$ [15], this is not expected if the measurement is taken in the periphery. In normotensive subjects, the diameter of elastic arteries (e.g. carotid artery) change linearly with pressure, while peripheral arteries (e.g. radial artery) exhibit saturation at near-systolic pressure [16]. In-vitro and in-vivo studies have demonstrated that the diameter-pressure relationship exhibits an exponential characteristic [8], [16], [17].

\section{B. Model 2 (M2): Exponential Relationship}

Many researchers have studied the exponential relationship between arterial diameter distension and BP [7]-[9], [18]. After obtaining the arterial vessel cross-section area, $A(t)$, from $D(t)$, the BP waveform can be calculated as follows:

$$
P(t)=P_{0} \cdot e^{\alpha\left(\frac{A(t)}{A_{d}}-1\right)},
$$

with

$$
\alpha=\frac{A_{d} \cdot \ln \left(P_{s} / P_{d}\right)}{A_{s}-A_{d}},
$$


where $\alpha$ is the vessel rigidity coefficient, $P_{0}$ and $P_{d}$ is the diastolic pressure, $P_{s}$ is the diastolic pressure, $A_{d}$ is the diastolic luminal area and $A_{s}$ the systolic luminal area.

As (3) is valid over a large pressure range [8], $\alpha$ is pressureindependent and constant. To accurately determine $\alpha$, it is worth noting that $P_{s}$ and $P_{d}$ must be obtained at the same position as $D(t)$. If the pressures are unknown, calibration to brachial sphygmomanometer could be done. Wang et al. [9] designed an ultrasonic device that uses the brachial calibration method with a measurement precision higher than $2 \mathrm{mmHg}$. However, it is known that $P_{s}$ changes drastically through the arterial tree due to arterial stiffening and wave reflection phenomenon. Moreover, brachial $P_{s}$ should not be used for calibration in other measuring sites, especially if the changes between systolic pressures are known to be very different as is the case between the brachial and radial artery [12].

In addition, an iterative correction of $\alpha$ was proposed by Meinders et al. [8]. For the first iteration, the rigidity coefficient, $\alpha_{i}$ with $i=1$, is calculated as indicated in (4), taking use of brachial $S B P$ as $P_{s}$. After determining $P_{i}(t)$, the measured mean pressure $\bar{P}_{i}(t)$ is compared to the $M A P$ determined at the brachial artery. If $\left|\bar{P}_{i}(t)-M A P\right|>0.01$ $\mathrm{mmHg}$, a new $\alpha_{i+1}$ is calculated until the mean pressure of the projected waveform converges to brachial $M A P$. The iterative $\alpha_{i+1}$ is calculated as follows:

$$
\alpha_{i+1}=\alpha_{i} \cdot \frac{M A P}{\bar{P}_{i}(t)} .
$$

\section{Model 3 (M3): Laplace's Law + Moens-Korteweg}

An US-based method for the assessment of the $P W_{B P}$ based on the integration of pressure was introduced by Vappou et al. [10]. The method relies on the measurement of local distension waveforms using US signals, together with the measurement of the local Pulse Wave Velocity (PWV) that leads to an estimation of the local stiffness. The theoretical principle combines Laplace's Law and the Moens-Korteweg (MK) equation.

Laplace's law,

$$
d P=\frac{E \cdot h \cdot d R}{R^{2}}
$$

relates an infinitesimal variation of the lumen radius $d R$ to the variation of internal fluid pressure $d P$, where $E$ is the Young's elastic modulus of the arterial wall, $h$ is the wall thickness and $R$ is the artery's luminal radius.

The MK equation relates the PWV to the elasticity of the arterial wall,

$$
P W V=\sqrt{\frac{E \cdot h}{2 \rho \cdot R}},
$$

where $\rho$ is the blood density.

By replacing the elasticity factor in (6) with $E$ calculated from (7), and integrating it over the cardiac cycle, the $P P$ waveform is given by

$$
\Delta P=2 \rho \cdot P W V^{2} \cdot \log \left(\frac{R(t)}{R_{0}}\right),
$$

with $\Delta P=P(t)-P_{0}$, and $P_{0}$ and $R_{0}$ corresponding to diastolic values of pressure and artery radius, respectively. As in most indirect BP measurement models, the methodology of Vappou et al. [10] only determines the $\Delta P$, maintaining $P_{0}$ unknown. A conversion to absolute $\mathrm{BP}$ measurement is done by adding the $D B P$ measured at the brachial artery to the $\Delta P$ calculated in (8).

In this study, a linear waveform calibration to brachial $D B P$ and $M A P$ was proposed. The calibration procedure takes the model's projected mean pressure $\bar{P}(t)$ (through integration over a cardiac cycle) and end-diastolic pressure $P_{\text {end }}$ to calculate the calibrating factor $m$ as

$$
m=\frac{M A P-D B P}{\bar{P}(t)-P_{\text {end }}} .
$$

The pressure waveform is then multiplied by the calibration factor and the slope intercept $b$ is added (calculated for $P(t)=M A P)$.

In the study with 11 subjects [10], good correlation $(0.94<r<0.98)$ was found between the $P P$ measured through radial tonometry using a commercially available system and the $P P$ obtained through the method described above. The Bland-Altman study [10] revealed a positive bias of $4.7 \mathrm{mmHg}$ and standard deviation of $4.45 \mathrm{mmHg}$.

\section{Model 4 (M4): Bramwell-Hill Equation}

Bramwell et al. [19] improved the MK equation such that the formula may be independent of Young's modulus $E$ and thickness $h$ of the vessel wall, directly relating PWV to the compliance $d A / d P$ as follows:

$$
P W V=\sqrt{\frac{A(t) \cdot d P}{\rho \cdot d A}} .
$$

By rearranging (10), and assuming that PWV remains relatively constant during a cardiac cycle, a pressure waveform can be expressed in terms of PWV and change in crosssectional area as [20]:

$$
P(t)-P_{0}=\rho \cdot P W V^{2} \cdot \ln \left(\frac{A(t)}{A_{0}}\right) .
$$

It should be noted that the PWV is dependent on the compliance and area of an artery and it should be determined at the local site of diameter distension measurement [20].

The same calibration procedure as in M3 was applied to this model.

\section{E. Model 5 (M5): Joukowsky's Equation + PWV}

Soleimani et al. [11] developed a system where the internal diameter and blood velocity waveforms were extracted from consecutive sonograms. The authors assume that blood is an incompressible fluid traveling through a flexible tube, and pressure can be determined through the fundamental water hammer phenomenon. Thus, water hammer principle can be projected to the arterial vascular system, where the opening and closing of cardiac valves correspond to mechanical valves in a hydraulic piping system. The water hammer equation, known as Joukowsky's equation, measures the change in 
pressure of a fluid resulting from a change in the fluid's velocity, taking into account its density and PWV,

$$
\Delta P=\rho \cdot P W V \cdot \Delta v,
$$

in which $\Delta P$ and $\Delta v$ are changes in pressure and velocity, respectively, relative to the initial value.

Solving (12) with (10), and applying the increment of pressure and area instead of its derivative, results in

$$
\Delta P=\rho \cdot(\Delta v)^{2} \cdot \frac{A(t)}{\Delta A},
$$

where, once again, relative $\Delta P$ is obtained instead of the absolute BP. The brachial $D B P$ is then added to the measured pressure and the absolute $P W_{B P}$ is determined. Soleimani et al. [11] developed a subject-specific calibration procedure based on the correlation between $\bar{P}(t)$ calculated from the proposed model and $\bar{P}(t)$ calculated from tonometry measurements at the radial artery. The calibrated model was validated on 20 male subjects via a standard sphygmomanometer, revealing a $P P$ correlation of 0.91 and mean absolute difference (through Bland-Altman analysis) of $1.333 \pm 6.548 \mathrm{mmHg}[11]$.

\section{F. Comparison between models}

It is worth noting the similarities between models M3 and M4. Both (8) and (11) are derived from the Bramwell-Hill equation that relates the MK equation to the Laplace's Law. However, when integrating over a cardiac cycle, Vappou et al. [10] proposed a model with the decimal logarithm, whilst the direct integration of the Bramwell-Hill equation applies the natural logarithm. Although it is expected that both models produce similar responses, the raw, non-calibrated BP values derived from (11) are higher than the ones obtained from (8). This changes after applying the corresponding $M A P$ and $D B P$ calibration, where both calibrated models exhibit the same curve. From here on, M3/4 refers to both calibrated models M3 and M4.

Table I summarizes the different model equations and the implemented calibration methods. All models are calibrated with $D B P$ and $M A P$. In $\mathrm{M} 2$, the first iteration is calibrated with $S B P$. Calibrating values $D B P, S B P$ and $M A P$ can be taken from a brachial sphygmomanometer measurement.

\section{Materials and Methods}

\section{A. Database}

For the benchmarking of the different $P W_{B P}$ models, the open-source simulated database of arterial PWs developed by Charlton et al. was used [13]. The computational model consists of three main components, based on the work of Alastruey et al. [21]. First, the arterial tree is decomposed into arterial segments modeled as thin viscoelastic tubes of constant length and linearly tapered diameter. Then, a periodic inflow waveform is introduced at the aortic root. Third, terminal three-element Windkessel boundary conditions are imposed at the outlets of peripheral arterial segments, modeling vascular beds. The database was validated by the comparison of PWs and hemodynamic characteristics with in-vivo data.

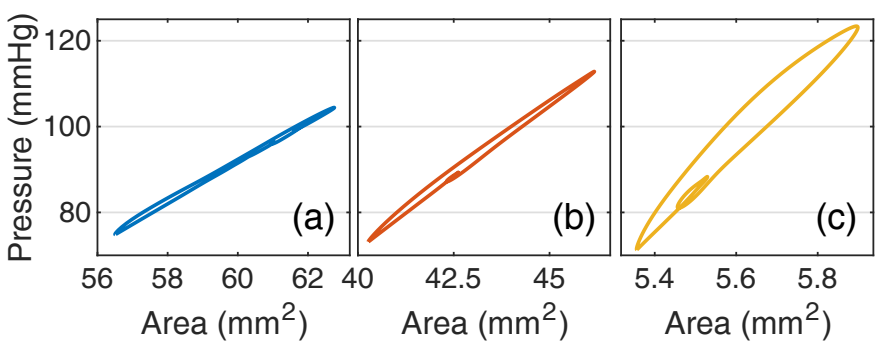

Fig. 1. Compliance curves $(\boldsymbol{P} / \boldsymbol{A})$ for (a) carotid, (b) brachial and (c) radial artery, extracted from the Charlton database [13] (for the baseline subject). The increase in hysteresis and $\boldsymbol{P P}$ towards the periphery due to an increase in stiffness is noticeable.

The primary parameters (flow velocity and luminal area) that can be directly extracted from ultrasound measurements were taken from the database, and then simulated in MATLAB for a comparative realistic analysis of the different five models. One of the most important simulations performed consisted of the compliance curves (arterial pressure versus luminal area), as it provided a valuable information about the viscoelasticity properties of the arteries. Fig. 1 shows the compliance curves derived from the baseline of a 25 year old virtual subject (one simulated subject created with the age-specific mean values for all variables, e.g. heart rate, stroke volume and arterial diameters). The viscoelasticity of the arterial walls leads to a hysteresis seen in the compliance curve. Furthermore, the composition of the arterial wall, in particular the elastin and collagen content, changes from central to peripheral arteries. Starting in the proximal aorta, elastin is the dominant component, but by the periphery collagen becomes the governing element [22]. As seen in Fig. 1, it is clear that there is a change in behavior and compliance through the arterial tree, so that the carotid artery presents an approximately linear relationship, whilst towards the periphery, in the radial artery, the hysteresis is much more accentuated due to the collagen content. Thus, when performing arterial parameters readings with ultrasound in the arterial tree (i.e. blood pressure measurements), changes through the different arteries are expected. Therefore, specific models or concrete stiffness coefficients should be applied to each local peripheral artery in the cardiovascular tree.

\section{B. Models simulation}

To evaluate and analyze the changes in the hemodynamic parameters in the arterial tree, the five BP models were studied at three main arteries: carotid, brachial and radial. For each measuring sites, luminal area PW, in combination with (i) PWV for M3 and M4, and (ii) flow velocity PW for M5, were extracted from the database and applied to each model. As $D B P$ and $M A P$ do not change significantly through the arterial tree, calibration was made to brachial $D B P$ and $M A P$ (additionally to $S B P$ in the case of M2). The aim of calibrating the curves to the brachial values is to approximate the simulation to a practical situation where calibration of the measured hemodynamic PWs and values would be done using a brachial sphygmomanometer. Furthermore, in the sense of maintaining real-life application plausibility, brachial $M A P$ was not calibrated to its gold standard - integration of BP curve 
TABLE I

MODEL EQUATIONS AND CALIBRATION PROCEDURE

\begin{tabular}{|c|c|c|c|c|}
\hline & Name & Equation & Calibration & Reference \\
\hline M1 & Linear Relationship & $P(t)=D(t) \cdot k+b$ & $k=\frac{M A P-D B P}{D(t)-D_{d}}$ & {$[8]$} \\
\hline M2 & Exponential Relationship & $P(t)=P_{0} \cdot e^{\alpha\left(\frac{A(t)}{A_{d}}-1\right)}$ & $\begin{array}{l}\alpha_{1} \text { with } P_{s}=S P B \text { and } P_{d}=D B P \\
\alpha_{i+1}=\alpha_{i} \cdot \frac{M A P}{\bar{P}_{i}(t)}\end{array}$ & {$[8]$} \\
\hline M3 & Laplace's Law + MK equation & $P(t)-P_{0}=2 \rho \cdot P W V^{2} \cdot \log \left(\frac{R(t)}{R_{0}}\right)$ & \multirow{2}{*}{$P(t)$ calibrated to $m=\frac{M A P-D B P}{\bar{P}(t)-P_{\text {end }}}$} & [10] \\
\hline M4 & Bramwell-Hill Equation & $P(t)-P_{0}=\rho \cdot P W V^{2} \cdot \ln \left(\frac{A(t)}{A_{0}}\right)$ & & [20] \\
\hline M5 & Joukowsky's Equation + PWV & $P(t)-P_{0}=\rho \cdot(\Delta v)^{2} \cdot \frac{A(t)}{\Delta A}$ & $\begin{array}{l}\text { correlation between M5 } \bar{P}(t) \text { and } \bar{P}(t) \\
\text { from tonometry measurements at the radial artery }\end{array}$ & {$[11]$} \\
\hline
\end{tabular}

$P_{0}$ calibrated to $D B P$. $D B P, S B P$ and $M A P$ calculated from brachial sphygmomanometer.

over a cardiac cycle - but through a mathematical equation that can be derived from brachial-cuff measurements. This correlates $S B P$ and $D B P$ to $M A P$. Hence, brachial $M A P$ was calculated as $M A P=0.42 \cdot S B P+0.58 \cdot D B P$ [23].

The process explained above was applied to 729 simulated 25 year old subjects available at the database. Next, the resulting $P P$ models were compared and correlated to the ground truth $P P$ waveforms $\left(P W_{P P}\right)$ supplied by the database $\left(P W_{P P}=P W_{B P}-D B P\right)$. Additionally, error boxplots for $D B P, S B P, P P, M A P$, and mean $P W_{B P}$ with its standard deviation $(S D)$ were calculated and compared to the database.

\section{Ex-vivo validation}

To determine the validity of the study, measurements with the most accurate model were performed in an ex-vivo setup (Fig. 2). The mimicked arm consisted of a porcine artery (inlet diameter $=3.67 \mathrm{~mm}$, outlet diameter $=5.13 \mathrm{~mm}$ ) submersed in saline solution $(\% \mathrm{w} / \mathrm{w}=0.9 \%)$. Pulsatile flow was established by a "heart-like" pump (Zentrifugalpumpe MultiFlow, GAMPT GmbH, Germany) at $51 / \mathrm{min}$. Two non-return valves at the inlet and outlet of the artery controlled the flow.

The distending diameter of the artery was measured through custom ultrasonic echo-wall tracking previously validated [24], and the derived pressure was compared to the pressure measured by a commercial pressure sensor (ABP Series, Honeywell International Inc). The custom-made $5 \mathrm{MHz}$ resonance-frequency US-probe (Fraunhofer IBMT, Germany) was driven by a general-purpose ultrasonic pulser/receiver (DPR300 Pulser/Receiver, Imaginant Inc., NY) with a pulse frequency rate of $2 \mathrm{kHz}$. For easier post-processing, the raw analog echo signal was filtered and the signal envelope of the received echoes was recorder through a new Low-Power Peak detector [24]. The signal envelope captured by the US, along with the pressure signals from the Honeywell-Sensor, were acquired by a PC-oscilloscope (Picoscope 5243D, Pico Technology Ltd., UK) at a sampling frequency of $500 \mathrm{MHz}$ and post-processed offline in MATLAB.

\section{Results}

\section{A. Models simulation}

BP curve of the baseline subject was calculated using the five models described above (Fig. 3). Note that after
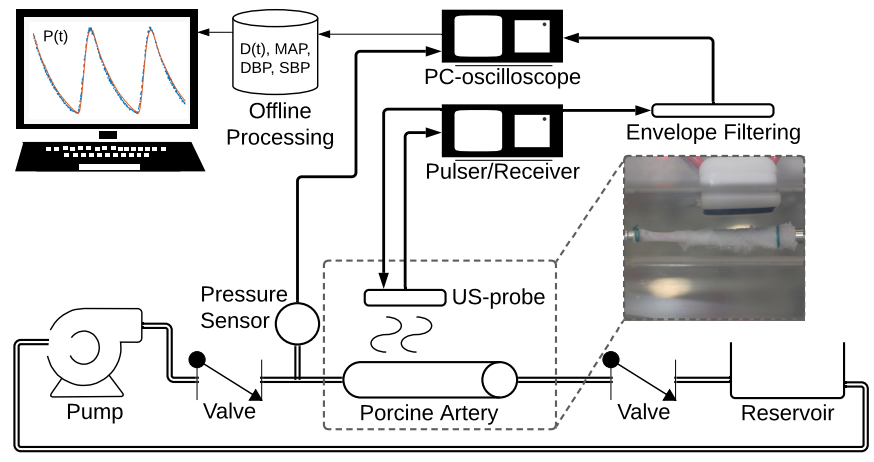

Fig. 2. Diagram of the setup for ex-vivo BP model validation. Doublelines represents the fluid path, thick arrows the analog signal and thin arrows the digital signal.

calibration, M3 and M4 exhibit the same waveform and thus only one curve is shown in the graph. Over-estimation of $S B P$ (and consequently, $P P$ ) was seen in M2 and M5. Except for M5, all PWs models were in good agreement with the pressure waveform except for a slight time delay. The waveform profile for M5 is not in accordance to the rest of the models, as it is modeled from the blood flow velocity profile, and not only from the arterial distension waveform. Due to the calibration procedure done with brachial $D B P$, under-estimation and over-estimation of $D B P$ is seen at the carotid artery and radial artery, respectively. An increase in time delay between ground truth and the models waveform is seen towards the periphery.

Pearson's linear correlation $r$ and mean difference $P P s$ obtained from the models were compared based on PPs extracted from the database (Table II). The study was done for 25 year old subjects $(n=729)$ at the carotid, brachial and radial artery. There is a remarkable improvement of the calibrated models in comparison to uncalibrated ( $\mathrm{M} X^{\prime}$ ) ones that is noticeable by the high increment of $r$. Notice that only uncalibrated M3', M4' and M5' were introduced, as M1 and M2 calibration procedure is intrinsic to the model. In all the measuring sites, M5 is always the least accurate, followed by M2. At the carotid artery, M3/4 exhibit a slightly better performance in $r$ and mean difference, whilst at the brachial and radial artery the $r$ is the same between models M1 and M3/4. Moreover, when comparing M1 and M3/4 at the radial artery, it is appreciated that for M1 the mean difference is slightly lower than M3/4. Summarizing, M1 is the best 


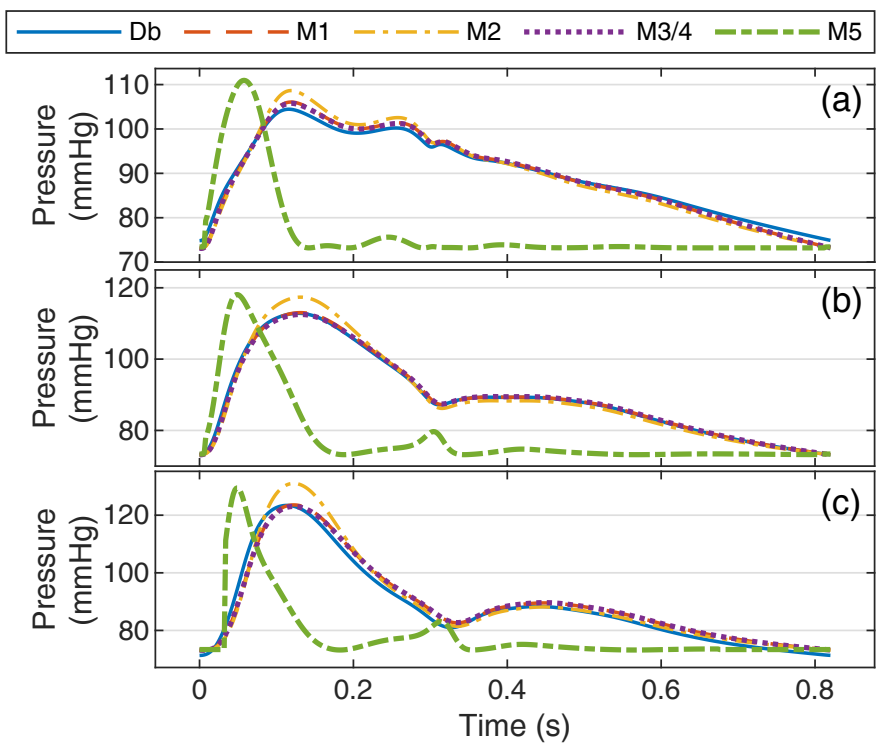

Fig. 3. BP models performance for the baseline subject at the (a) carotid, (b) brachial and (c) radial artery. All models are calibrated as described in section III. Notice that M1 and M3/M4 curves overlap. Curve $D b$ refers to the database pressure waveform (ground truth).

performing model at the radial artery, revealing a correlation of 0.978 and mean difference of $(-2.134 \pm 2.477) \mathrm{mmHg}$.

After rejecting M5 because of its high error and low correlation with the $P P$ extracted from the database, further error studies between the best performing models were done. $D B P$ and $M A P$ display the same error profile between the remaining models, since the models are calibrated from the same values (brachial $D B P$ and $M A P$ ). The results of the error studies for 729 subjects are depicted in the boxplots in Fig. 4, where mean $P W$ error, its $S D$ and $S B P$ error are included. Even though M2 always exhibits a lower mean $P W$ error (carotid artery (c.a.) $=-0.313 \mathrm{mmHg}$, brachial artery (b.a.) $=0.013 \mathrm{mmHg}, \quad$ radial artery (r.a.) $=1.160 \mathrm{mmHg}$ ), the $S D$ (i.e. r.a. $=2.864 \mathrm{mmHg}$ ) and SBP error (i.e. r.a. $=7.301 \mathrm{mmHg}$ ) are higher. The mean PW error is the same in M1 and M3/4 at all sites (c.a. $=-0.318 \mathrm{mmHg}$, b.a. $=0.023 \mathrm{mmHg}$, r.a. $=1.170 \mathrm{mmHg}$ ). In the carotid artery, M3/4 has a smaller $S D$ and $S B P$ error $(1.002 \mathrm{mmHg}$ and $1.252 \mathrm{mmHg}$, respectively) than $\mathrm{M} 1$ $(1.008 \mathrm{mmHg}$ and $1.505 \mathrm{mmHg}$, respectively). However, at the brachial and radial artery $\mathrm{M} 1$ has a lower $S D$ (b.a. $=0.658 \mathrm{mmHg}, \quad$ r.a. $=2.013 \mathrm{mmHg}$ ) and $S B P$ error (b.a. $=0.135 \mathrm{mmHg}$, r.a. $=-0.151 \mathrm{mmHg}$ ) than $\mathrm{M} 3 / 4$ (i.e. SBP error: b.a. $=-0.345 \mathrm{mmHg}$, r.a. $=-0.657 \mathrm{mmHg}$ ). Thus, it is corroborated that in a 729 subject-study, M1 is the most accurate model for the radial artery.

\section{B. Ex-vivo validation}

For the ex-vivo validation, the linear model (M1) was selected as the best performing model and applied to the setup. Fig. 5 shows the results of the commercial pressure sensor measurements and the curve when applying M1 to the distension measurements acquired by the custom fabricated ultrasound sensor. The Pearson's linear correlation between

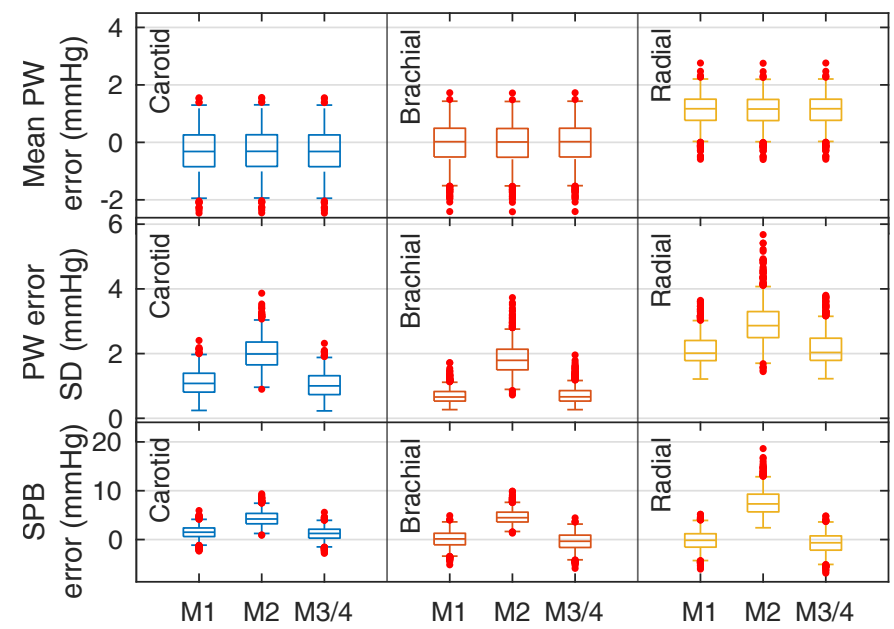

Fig. 4. BP models error $(n=729)$ at the carotid, brachial and radial artery for the best models (M1, M2, M3/4). First and second row illustrates mean pulse wave error and its standard deviation. SBP error is shown in third row.

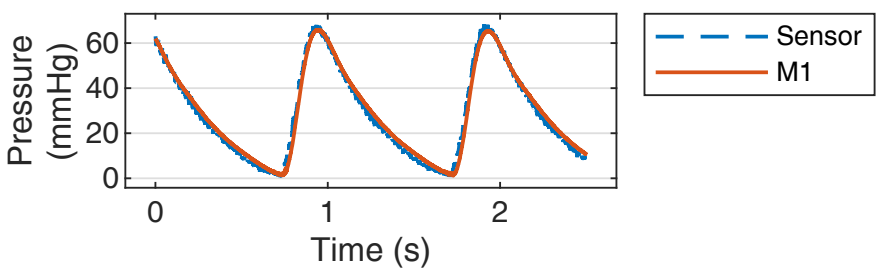

Fig. 5. Pressure waveforms obtained by the commercial pressure sensor and linear model (M1) during 2.5 seconds. Pump flow was set to $5 \mathrm{l} / \mathrm{min}$. Pulse repetition frequency at $2 \mathrm{kHz}$.

the obtained $P W_{B P}$ when applying $\mathrm{M} 1$ to the ultrasound measurement and the commercial pressure sensor was of $r=0.994$ and mean difference of $(0.544 \pm 2.315) \mathrm{mmHg}$. The SBP error was of $-2.131 \mathrm{mmHg}$.

\section{DISCUSSION}

US-based methods are feasible in most vascular sites and cost effective, being promising as indirect, non-invasively, continuous, and comfortable means of obtaining arterial pressure waveforms, and providing anatomical and functional information about arterial health. Several models have been proposed that relate hemodynamic variables indirectly to BP. In the present work, five models that ultimately take the distending luminal area were studied in-silico through an arterial PW simulated database. To the best of our knowledge, this was the first time a comparison study of BP models was done in a large $(n=729)$ and variable database.

All the studied models rely on the physical assumptions that the luminal area is a circle, neglecting the possibility of irregular and asymmetric area and, therefore, excluding the cases of highly torturous arteries. Additionally, the models assume linear elasticity, meaning that pressure and luminal area PWs have the same temporal profile. However, due to the natural viscoelasticity of arteries, this is known not to be true and it is corroborated in this study with the compliance curves and the time delay between the ground truth pressure and the calculated $P W_{B P}$ from the different models. The time delay 
TABLE II

PEARson's LiNEAR CORRELATION AND MEAN DIFFERENCE BETWEen PP MODELS. Ground truth PP (N=729) AT the CAROTID, BRACHIAL AND RADIAL ARTERY.

\begin{tabular}{|c|c|c|c|c|c|c|}
\hline & \multicolumn{2}{|r|}{ Carotid artery } & \multicolumn{2}{|r|}{ Brachial artery } & \multicolumn{2}{|r|}{ Radial artery } \\
\hline & $r$-Pearson & Mean difference $(\mathrm{mmHg})$ & $r$-Pearson & Mean difference $(\mathrm{mmHg})$ & $r$-Pearson & Mean difference $(\mathrm{mmHg})$ \\
\hline M1 & 0.979 & $3.459 \pm 1.560$ & 0.989 & $\mathbf{0 . 0 8 0} \pm 1.719$ & 0.978 & $-2.134 \pm 2.477$ \\
\hline M2 & 0.970 & $6.317 \pm 1.887$ & 0.985 & $4.665 \pm 1.511$ & 0.969 & $5.661 \pm 2.837$ \\
\hline M3' & 0.571 & $-17.758 \pm 6.233$ & 0.485 & $-23.578 \pm 7.545$ & 0.818 & $-30.665 \pm 7.315$ \\
\hline M3 & 0.980 & $3.167 \pm 1.558$ & 0.989 & $-0.428 \pm 1.806$ & 0.978 & $-2.655 \pm 2.572$ \\
\hline M4' & 0.571 & $-1.475 \pm 6.238$ & 0.485 & $-1.907 \pm 8.053$ & 0.818 & $-2.032 \pm 5.810$ \\
\hline M4 & 0.980 & $3.167 \pm 1.558$ & 0.989 & $-0.428 \pm 1.806$ & 0.978 & $-2.655 \pm 2.572$ \\
\hline M5 & 0.917 & $-12.179 \pm 3.457$ & 0.812 & $-14.525 \pm 5.061$ & 0.490 & $-16.318 \pm 8.985$ \\
\hline M5 & 0.917 & $7.791 \pm 3.459$ & 0.812 & $5.529 \pm 5.059$ & 0.485 & $3.853 \pm 9.030$ \\
\hline
\end{tabular}

is larger at the radial artery, due to the increase in collagen content and, consequently, viscoelasticity.

Another common limitation between the models is that all non-invasively acquired arterial pressure waveform require calibration to a known BP, imposing potential sources of measurement errors. It is generally accepted that $D B P$ and $M A P$ do not change considerably through the arterial tree, however, there are small changes, as seen in this study, introducing overand under-estimation in $D B P$ at the carotid and radial artery, respectively, when calibrated to brachial $D B P$. Furthermore, it is known that every formula to calculate $M A P$ that uses a form factor $(F F)$ that quantifies the change in the shape of $P W_{B P}$ and relates its mean pressure to $S B P$ and $D B P$ have flaws. As the shape of BP changes along the artery tree due to propagation and reflection phenomena, it is understandable that a common $M A P$ equation is not possible. Whilst a $F F$ of 0.3 might be closest to the invasive measured $M A P$ at the radial artery, the same could not be said about the brachial artery. A study by Papaionnou et al. [23] demonstrated that the estimation of $M A P$ using the $F F$ value 0.412 provide results that are more accurate to invasive brachial $M A P$ calculation when compared with $M A P$ determined by the traditional formula, which uses $F F=0.3$. In the current work, $F F=0.412$ was used to best approximate brachial $M A P$ to its true value.

The M5 pressure shape shown in Fig. 3 converges to the one displayed by Soleimani et al. [11], which is characterized by a distinct and separate two-peak waveform. All models except for M5 were able to approximate the shape of their BP waveform to the ground truth. The differences between the shapes can be appointed to M5 taking the flow velocity profile into account, while for the rest of the models the only time dependent variable is the change in luminal area and its shape is similar to the pressure PW. Additionally, it is worth noting that the calibration procedure proposed by the authors, that correlates a subject $M A P$ values from its proposed method and values measured from the radial artery by tonometry over a group of cardiac cycles, could not be applied in the current study, as the database only supplies one cardiac cycle per subject. This underlying impracticability may be the subject of an increased $P W_{B P}$ error, as the subject-specific calibration was done with the values supplied by Soleimani et al. [11].

The simulation study showed a slight difference in the best applicable model (M1) at different measuring sites. Thus,
M1 attributes to each artery distinct composition and, consequently, specific physical behavior. However, while in theory the carotid artery exhibits a more linear compliance behavior than the radial artery, M3/4 (logarithmic model) proved to have a lower error value than M1 (linear model). The contrary was seen at the radial artery, where although there is a stronger viscoeleasticity and arterial rigidity, M1 turned out to be the model with the lowest error. Nonetheless, the differences in error between $\mathrm{M} 1$ and $\mathrm{M} 3 / 4$ are quite small $(<0.6 \mathrm{mmHg})$ and may be neglected.

The Association for the Advancement of Medical Instrumentation (AAMI) recommends a maximum mean difference of $\pm 5 \mathrm{mmHg}$ and standard deviation of $\pm 8 \mathrm{mmHg}$ between the standard and the novel method of pressure measurement (AAMI/ISO 81060-2:2013) [25]. Taking the database's $P W_{B P}$ as standard, M1 and M3/4 accomplished this requirement, whilst M2 and M5 introduced higher mean difference and variability $((5.661 \pm 2.837) \mathrm{mmHg}$ for $\mathrm{M} 2$ and $(3.853 \pm 9.030) \mathrm{mmHg}$ for M5, when applied at the radial artery), not complying to the recommendations. As the exponential relationship is the most used model for extracting pressure waveform from ultrasound measurements of arterial wall distensibility in known literature review [7]-[9], it was expected that M2 would reveal a better accuracy than M1 (linear model) but that was not the case. Whilst the linear model (M1) follows AAMI recommendations and has the best results in the brachial and radial artery, the exponential model (M2) metrics are above the recommended values and is the second worst performing model in the present study.

The ex-vivo study revealed a better correlation $(r=0.994)$ than in-silico $(r=0.978)$. This may be due to the fact that the models calibration in ex-vivo was done by using the in site pressure sensor's $D B P$ and $M A P$ recording. Thus, the calibration was done specifically to the measured artery (radial) and not to the brachial artery, which increases the error for comparison with realistic and practical measurements.

\section{CONCLUSION}

The present study provides a novel in-silico analytical comparison between different models that relate arterial pulse waves to the pressure waveform for a continuous, non-invasive monitoring of arterial health parameters. The best model for the radial artery has been validated with an ex-vivo experiment, in which a porcine artery and an ultrasound sensor were used. 
The ex-vivo experimental measurements demonstrated the high accuracy of the linear model based on the MAP calibration with extremely low error values of $(0.544 \pm 2.315) \mathrm{mmHg}$. This research introduces a new insight into the extraction of arterial parameters based on ultrasound, that will help the researchers to get much more precise clinical non-invasive readings. The changes in the pressure waveform through the arterial tree makes it necessary a site-specific model calibration method for a high accurate non-invasive sensor measurement.

\section{REFERENCES}

[1] Hypertension. World health organization (2019). [Online]. Available: https://www.who.int/news-room/fact-sheets/detail/hypertension

[2] T. Unger et al., "2020 international society of hypertension global hypertension practice guidelines," Hypertension, vol. 75, no. 6, pp. 1334-1357, Jun. 2020.

[3] L. Peter, N. Noury, and M. Cerny, "A review of methods for non-invasive and continuous blood pressure monitoring: Pulse transit time method is promising?" IRBM, vol. 35, no. 5, pp. 271-282, Oct. 2014.

[4] R. Mukherjee et al., "A literature review on current and proposed technologies of noninvasive blood pressure measurement," Telemedicine and e-Health, vol. 24, no. 3, pp. 185-193, Mar. 2018.

[5] T. Arakawa, "Recent research and developing trends of wearable sensors for detecting blood pressure," Sensors, vol. 18, no. 9, p. 2772, Aug. 2018.

[6] C. Peng et al., "Noninvasive and nonocclusive blood pressure monitoring via a flexible piezo-composite ultrasonic sensor," IEEE Sensors Journal, vol. 21, no. 3, pp. 2642-2650, Feb. 2021.

[7] B. Gavish and J. L. Izzo, "Arterial stiffness: Going a step beyond," American Journal of Hypertension, vol. 29, no. 11, pp. 1223-1233, Jul. 2016.

[8] J. M. Meinders and A. P. Hoeks, "Simultaneous assessment of diameter and pressure waveforms in the carotid artery," Ultrasound in Medicine \& Biology, vol. 30, no. 2, pp. 147-154, Feb. 2004.

[9] C. Wang et al., "Monitoring of the central blood pressure waveform via a conformal ultrasonic device," Nature Biomedical Engineering, vol. 2, no. 9, pp. 687-695, Sep. 2018.

[10] J. Vappou et al., "Pulse wave ultrasound manometry (PWUM): Measuring central blood pressure non-invasively," in 2011 IEEE International Ultrasonics Symposium. IEEE, Oct. 2011.

[11] E. Soleimani et al., "Assessing the blood pressure waveform of the carotid artery using an ultrasound image processing method," Ultrasonography, vol. 36, no. 2, pp. 144-152, Apr. 2017.

[12] M. K. Armstrong et al., "Brachial and radial systolic blood pressure are not the same," Hypertension, vol. 73, no. 5, pp. 1036-1041, May 2019.

[13] P. H. Charlton et al., "Modeling arterial pulse waves in healthy aging: a database for in silico evaluation of hemodynamics and pulse wave indexes," American Journal of Physiology-Heart and Circulatory Physiology, vol. 317, no. 5, pp. H1062-H1085, Nov. 2019.

[14] X. Ding and Y.-T. Zhang, "Pulse transit time technique for cuffless unobtrusive blood pressure measurement: from theory to algorithm," Biomedical Engineering Letters, vol. 9, no. 1, pp. 37-52, Feb. 2019.

[15] L. M. V. Bortel et al., "Non-invasive assessment of local arterial pulse pressure: comparison of applanation tonometry and echo-tracking," Journal of Hypertension, vol. 19, no. 6, pp. 1037-1044, Jun. 2001.

[16] A. P. G. Hoeks, "Non-invasive study of the local mechanical arterial characteristics in humans," in Developments in Cardiovascular Medicine. Springer Netherlands, 1993, pp. 119-134.

[17] C.-K. Sun, "Cardio-ankle vascular index (CAVI) as an indicator of arterial stiffness," Integrated Blood Pressure Control, p. 27, Apr. 2013.

[18] M. Kachuee et al., "Cuffless blood pressure estimation algorithms for continuous health-care monitoring," IEEE Transactions on Biomedical Engineering, vol. 64, no. 4, pp. 859-869, Apr. 2017.

[19] J. C. Bramwell and A. V. Hill, "The velocity of pulse wave in man," Proceedings of the Royal Society of London. Series B, Containing Papers of a Biological Character, vol. 93, no. 652, pp. 298-306, Apr. 1922.

[20] J. Seo et al., "Noninvasive arterial blood pressure waveform monitoring using two- element ultrasound system," IEEE Transactions on Ultrasonics, Ferroelectrics, and Frequency Control, vol. 62, no. 4, pp. 776-784, Apr. 2015.

[21] J. Alastruey, K. Parker, and S. Sherwin, "Arterial pulse wave haemodynamics," BHR Group - 11th International Conferences on Pressure Surges, pp. 401-442, 102012.
[22] G. J. et al., "Ultrasonography and tonometry for the assessment of human arterial stiffness," in Applied Aspects of Ultrasonography in Humans. InTech, Apr. 2012.

[23] T. G. Papaioannou et al., "Mean arterial pressure values calculated using seven different methods and their associations with target organ deterioration in a single-center study of 1878 individuals," Hypertension Research, vol. 39, no. 9, pp. 640-647, May 2016.

[24] A. B. Amado-Rey et al., "Extraction of radial-artery strain and stiffness by using noninvasive ultrasound and a low-power peak detector," IEEE Sensors Letters, vol. 5, no. 8, pp. 1-4, Aug. 2021.

[25] A. N. S. Institute. Non-invasive sphygmomanometers - part 2: clinical investigation of automated measurement type (ansi/aami/iso 81060-2:2013). [Online]. Available: http://webstore.ansi.org

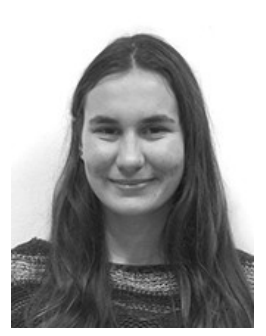

Ana Carolina Gonçalves Seabra received the B. S. degree in biomedical engineering from University of Minho (UMinho), Braga, Portugal in 2019 and is currently pursuing the M.S. degree in medical electronics at Uminho in corporation with the University of Freiburg, Freiburg im Breisgau, Germany.

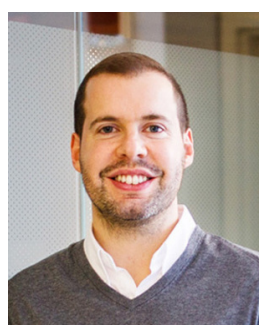

André Ferreira da Silva received the M.Sc. degree in Biomedical Engineering (2007) and the $\mathrm{Ph}$.D. degree in Leaders for Technical Industries (2011) from the University of Minho (UMinho), Braga, Portugal. From 2011-2021 was an Invited Assistant Professor at UMinho and between 2018-2021 the Executive Coordinator of the MIT Portugal Program. Presently, he is an Assistant professor for the Department of Industrial Electronics at UMinho for the disciplinary area of Electronic Instrumentation and Microsystems.

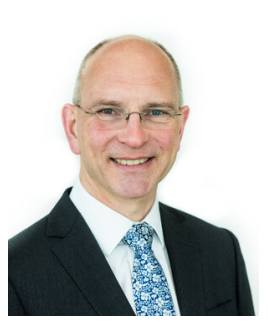

Thomas Stieglitz (SM) received the Diploma degree (1993) in electrical engineering from Technische Hochschule Karlsruhe (now KIT), Germany, and the Ph.D. (1998) and Habilitation (2002) degrees from Saarland University, Saarbrücken, Germany. Since 2004, he has been a Full Professor of Biomedical Microtechnology with the Department of Microsystems Engineering , University of Freiburg, Freiburg, Germany. He has coauthored about 160 peerreviewed journal publications and 330 conference proceedings and filed about 30 patents. His research interests include neural implants, monitoring of biological signals and electrical stimulation.

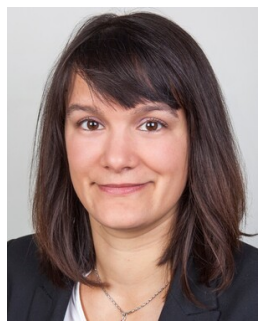

Ana Belén Amado-Rey received the Dipl.-Ing. degree (2010) and Master's degree (2014) in telecommunication engineering from the University of Extremadura, Caceres. From 2014-2018, she was at Fraunhofer IAF, Freiburg, where she was involved with GaAs mHEMT technology. After her PhD (2018), she joined the laboratory of biomedical microtechnology at the University of Freiburg. Her main research focus on the PCB electronic development at ultrasound frequencies and piezo characterization. 